\title{
Play-Driven Design: Model for Engaging Personas in Content Sites
}

\author{
Angelo Públio ${ }^{1}$, Sergio Nesteriuk ${ }^{2}$ \\ ${ }^{1,2}$ Escola de Ciências Exatas, Arquitetura e Design. Universidade Anhembi Morumbi, Brazil
}

\begin{abstract}
Play-Driven Design (PDD) is a design model aimed at drawing the user's attention to a content website, involving them and leading them to spontaneous creation of content. For the PDD to provide meaningful experiences and engage personas, this text addresses some game design elements, suggesting an iterative application and an evolutionary perspective of web design projects. When applied, the model boosts new approaches for the site to connect with the user.
\end{abstract}

Keywords: Design, play, personas, engaging, content sites, Play-Driven Design

\section{Introduction}

If on the one hand we are experiencing a time of information overload [1], on the other, we are always trying to find content on the web. It is the so-called "information seeking" [2]. In addition to the search on their own initiative, users may also learn about the existence of certain content through, say, an e-mail received or an update on social networks - either accessing the display of this content or being redirected to some site. Websites do not always manage to hold the attention of the public, often due to lack of focus and diffuse thinking in the face of everyday tasks, contaminated by the multiple occurrences of noise around us [3]. Amid this profusion of information, how can one awaken a user and drag their attention to a particular website, involving and leading them to spontaneous creation of new content?

The purpose of this study is to propose a new user-centered design model [4] applied to content websites, which becomes the source of an experience of playful interaction and engagement. With that in mind, a gameplay dynamics is proposed, based on dramatic play. Content websites are understood as those meant to be a reference for users while in information seeking and gameplay, the set of necessary strategies to reach specific outcomes [5].

The proposal of the Play-Driven Design (PDD) model is to define a process that promotes engagement based on personas, so that a content website is not only a static repository of information, but also allows the generation of new, spontaneous content by its users, based on the game dynamics, allowing a more playful interaction between site users and maintainers.

\section{Methods}

As methodology and procedures, an exploratory research was used, through a bibliographical survey and also an action research in a content website, which became a case study - the researcher's personal website (https://angelopublio.com.br). The choice of this site is justified by the autonomy in carrying out the necessary tests and adjustments during the application of the action research.

Combined with an e-mail and social network asynchronous communication, interested websites can apply PDD as a way to stimulate user engagement. With the aid of play-tuning strategies, one may identify improvements that promote a more meaningful user experience and direct it to play.

Considering the PDD application that allows meaningful play on a website, users should have the ability to interact and deal with the website just as players do with a game. Thus, they can create an environment with intrinsic dynamics that benefits users interested in accessing information. The engagement of users in the PDD model is based on the principle of games and in the establishment of communities. The model is not intended to determine how identification and dynamics for engagement can occur, as this will depend on the hindrances and resources available for each project in which the model is applied. However, in detailing it, an identification of personas is exemplified, as well as a dynamic of engagement that can guide other projects.

In action research, a user could browse the website along existing content or submit their inquiry via a form. From the acceptance of the question by the moderator, it would be published on the website, visible to visitors and shared on social networks. Asynchronous communication becomes useful, for example, to encourage discussion on an ongoing basis, usually of more in-depth and reflexive [6] character.

Once new content, such as questions and answers, is posted on the website, it can be found by web crawler softwares. Thus, users' actions may contribute for new users to access the website as they look for information through search engines or while spontaneously sharing website content on social networks. 


\section{Result}

The result is a design (canvas) model proposed, a confluence between play, game design and game dynamics, supported by content, dramatic game dynamics, web design and technology. PDD is organized in a cyclical way, which allows the constant improvement of its structural model. The expected sequence of the three stages is: identifying; engaging; delivering. The optimization stage is common to all three other stages of the model, not just at the end of a cycle, but during each and every stage, since each of them can only occur if the previous stage is settled. All stages have equal importance in the PDD process to ensure meaningful play to users. Let's probe each stage:

1) Identify, responsible for the identification of personas. Once the user logs on to the system, the information may be saved in a cookie so that there is no need for it to be requested again in the future. Cookie solutions and forms in which the user can directly insert their name and e-mail, without having to create a user and password or to authenticate to the website, are elements that help to keep the client aware and focused on the task performed.

2) Engage, in which the engagement of personas happens, with the site structure planned to receive users' actions and promote meaningful play. The term "mission path" will be used in references to the set of rules, diagram, actions expected of users, feedbacks, mechanisms, and site elements that comprise a common goal.

3) Deliver, in which the system seeks for user feedback and for conveying the impression that it has control over the system (one of the components of the flow [3]).

4) Optimize, based on the concept of game tuning, on data collection of user behavior on the website, on emails sent by the system, on usability testing (e.g. heat map) and on the online user evaluation. With that in hand, the website designer will have enough data to make the necessary adjustments for the continuous improvements in the system, within the logic of an iterative process.

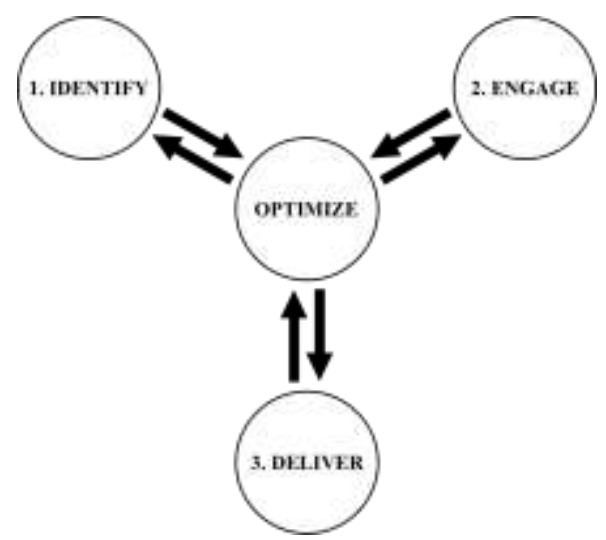

Figure 1 - Three-stage cyclical process of Play-Driven Design: identify, engage, deliver, with the optimizing phase present in all of them.

\section{Discussion}

For Huizinga [7], the play is a fact that precedes our society and even men themselves. Humans and other animals as well get involved in a play carried by the playful instinct. In the adult human, this occurs on a voluntary basis, characterizing an act of freedom. Still according to Huizinga, the play is capable of transporting the player from his "real" life to the game world, with own rules, dynamics and values. The boundary that defines the limits between these two realities is what Huizinga called the "magic circle" [8]. This feature, which could question the seriousness of play, creates the opposite effect in that it completely involves the player. There is no resistance to the imaginary world that is created within the magic circle, as the player is in "suspension of disbelief" [5].

The play that takes the player to this "break" in life has limits, both of time and space. From the beginning to what is established as the end of the game, everything that encourages the player is a set of associations with own senses, which creates a new memory and, even after its end, can be repeated when played again. Salen e Zimmerman [9] call "space of possibilities" the journey that begins in the same place but offers a varied experiential path at each game with different results, even when the formal structure of the game and its rules remain the same - which is a reason for this concept to be referred to as "equal, but different".

Players share relevant experiences and this creates bonds, which often go beyond the game itself. Thus, within the scope of the PDD, the search for information in content websites may also prove to be important experiences for its users. If a content website allows engagement among users, it can also promote a link between them, enabling community building, as is seen in games. According to Caillois [10], the game is related 
to the activity itself, but also to the elements necessary for its accomplishment. The game practice requires precision, since the absence or existence of an element can jeopardize it unless such flexibility is tolerated and is part of its rules. The game transmits freedom, in the condition of an activity carried out spontaneously. Meanwhile, it is linked to joy and fun, and is also guided by luck, intelligence or strategy.

As an isolated activity, the game would quickly lose its charm. As individual as a game may be, the existence of three elements, even if in an imaginary way, is capable of prolonging the fun: spectators, rivalry, and location [10]. The habit of the group meeting in a certain place for the practice of a game ends up consecrating and delimiting that space. Over time, its members end up setting rules with common consent. Approaching people with common interests is relevant to complement the pleasure provided by the gaming experience.

The existence of a community inspires each player individually, in that it encourages players to excel and relate. The game, with its capacity to give satisfaction and pleasure, can reach life and even become an obsession (pathological game), except when the game is a profession for the player. As players perform an action, they are "adding content" to the game [11]. An example of undeclared content in the rules, but emerging is the bluff in a poker game [9]. A game that can be guided by strategies and defined by its rules, such as a board game, is considered as an emergent game. A step-by-step game of what must be done at each stage is characterized as a game of progression, very common, for example, in adventure games. While some games may be defined specifically as emergent or progressive, there are cases that can be treated as hybrid, in which a series of objects emerge in the game, but the player must perform a specific sequence of events to complete an action [12].

An emergent system uses unpredictability as a way to surprise the player. However, the fact that the game makes use of unpredictability does not guarantee per se that it will fulfill the role of challenging the player [12]. The opposite may also occur: a player experienced in tic-tac-toe cannot consider a match won in its beginning, since a match exceeds 50,000 gaming possibilities [9]. A game design model that can generate an unexpected gameplay while maintaining simple choices is triangularity in the "rock-paper-scissors" game, when it builds an intransitive relationship between these three units, once every unit has its strength and weakness [12]. The progression stimulus is maintained in the player as a response from the system is received as a reaction to their action following a cyclic scheme of stimulus-response, action-reaction.

Competition and cooperation are part of animal nature, whether to define the status of a member in the community or for survival purposes. Competition is also a way of knowing each other's abilities and how each individual can cooperate for the development of the whole team [16]. When players share the same goals in games, social aspects of competition and cooperation are involved in the play [13]. Multiplayer group games are strategically planned to promote interactions (cooperation) among their members. Therefore, they have in communication an important ally in the game strategy on the way to victory (competition). The creation of communities is a way for players to discuss strategies, but also to socialize, becoming closer [12]. The creation of communities related to a game is in keeping with the ideas presented by Huizinga [7] and Caillois [10], in which players who share the same interest come together for the practice of the game.

Schell [14] highlights some aspects that should be considered in order to obtain a meaningful play by cooperation and competition in the game: determine the most skilled players, considering that there is a certain human compulsion in demonstrating superiority; allow communication between players, since that is an issue that inspires cooperation; provide feedback when the team succeeds, as a way to create more lasting social bonds.

Csikszentmihalyi [15] has determined that it is possible to attain pleasure with an intensely accomplished activity, in which we feel we are in control of the situation and mastering our own destiny. With this, we reach the flow state and an optimal experience, which is exactly what game designers wish to provide with their creations [15]. Optimal experience is the term Csikszentmihalyi uses to describe an extreme feeling of joy and pleasure that remains and becomes a kind of landmark in our lives even after the activity that caused it has ceased.

The relationship created and maintained between the player and his goal is the central component of pleasure in the game. Thus, the game designer has great influence on the modeling of the play experience, once it is he who establishes mechanisms and purpose of the game that are accepted by the players. The game has a macro-level end goal that the player pursues, but with a long-delayed outcome. If, during this long path, players do not perceive accomplishment and progress, their play experience will be threatened. That is how short-term goals can move the player, giving a more immediate feeling of exploration and control in the game. [9]

As players perform an action in the game, they must understand that this action has been recorded and will generate a consequence in the system [16]. The information they receive during this interaction is generally considered feedback, but it also comprises the change that their action causes on another element of the system [17]. When receiving some feedback, preferably immediate and easy to understand, a player better apprehends how to play, reducing their insecurity and receiving support [16]. 
The way the player's action reflects in another action can be understood from three central elements that constitute a cybernetic system [9]: sensor, which monitors the state of the system or environment; comparator, which decides whether an action needs to be taken based on the sensor information; and activator, which activates an action based on the comparator's decision.

PDD is a way of conceiving the design of a content website so that it takes advantage of game design, showing that there is a modus operandi in games that can be used in content creation, elaborating aspects of games on a website without necessarily transforming it in a game. In a user experience design (UX design), [18], it is common to resort to an archetype that brings together a group of users with common characteristics and a same goal. With the segmentation of users, it becomes possible to prioritize the needs of a group and what each individual seeks to achieve [4]. For better use of the proposed model, it is fundamental to identify the user persona, which can occur directly or indirectly. The direct form is given by an element present in the website interface with this purpose, such as fields in a fill-out form. The indirect way is the possibility of identifying the persona of a user based on the history of content browsed in the site.

The election of content sites for getting a playful-oriented design lies in the fact that this type of site has features that can contribute to its approach to the virtual worlds known as MUDs. Virtual worlds are implemented by computer networks in which people's actions may manifest as emergent behavior and simultaneously affect this shared or multiuser environment [19]. From the reading of Bartle [19] on MUDs and the playful interaction of players in communities, the possibility of transporting such engagement to the content website environment was perceived.

According to Bartle [19], one of the main elements for immersion is familiarity, which is often associated with the similarity between a virtual graphic environment and the immediate reality of the user. However, while the graphic elements lead the user to an immersion through the senses, in a textual project, immersion happens mostly through cognition and imagination. The written text can reach another form of immersion and cover nuances beyond the graphs and our sensitive reality.

The website used as an object of study is targeted at digital marketing professionals who are looking for information on inbound marketing - a digital marketing platform that centralizes all marketing actions (blogs, website pages and social networks). The theme was initially addressed by Halligan and Shah [20], and has as a principle the frequent creation and distribution of content through the regular production of new texts. Thus, the websites are indexed by the search systems, publicized in the social networks and by e-mail to registered users, giving more visibility and consequently generating new visits.

During the inbound marketing planning on a website, buyer personas are mapped, which defines the archetypes in terms of their demographic characteristics and those related to the consumption process. The contents created are aimed at offering information to buyer personas while perceiving their maturity as they make a purchase; that comprises three stages of the Buyer Journey: awareness, in which the consumer needs information to identify a problem; consideration, when the problem is defined and one seeks to identify possible solutions; and decision, in which the consumer is interested in content that helps evaluate the options selected to solve their problem.

The inbound method usually publishes on the website contents produced by copywriters (fixed or outsourced) or guests. Many authors look for blogs as a way to promote their website by publishing hyperlinked texts. Since the website analyzed was not a source of revenue, content production would depend almost exclusively on the researcher or on invited authors. Small entrepreneurs and startups often have resource limitations, and the deriving difficulty to maintain the frequency of content creation - even knowing the importance of visibility on the internet. This limitation has led to the reflection of how it would be possible to stimulate a spontaneous production of content by the users themselves. The studies carried out sought to identify a feasible model, able to create a form of frank relationship with users.

For a content website to promote engagement and direct the user to meaningful play, new features must be incorporated, as well as forms and the remittance of e-mails. PDD's main contribution is related to the design methodology, so designers can contemplate the website objectively, as a means for the users' engagement. When adopting this model, the websites will be primarily prioritizing the user's pleasurable experience.

The version of the website under study was developed on WordPress platform, the one used by $26.1 \%$ of all Internet sites [21]. That gives a broad perspective of technology replication. Commercial plug-ins were also used, which allowed data input and the remittance of questions by the users. Upon approval for publication, a question had an image associated, which increased visibility in social networks. In addition, an image in the page content contributes to the indexation and positioning of the new page in the search systems.

The function of a marketing automation software within the PDD is to carry out a workflow of e-mail remittance to the contacts previously registered. Triggering can occur when information is submitted on the website, sent simultaneously to the marketing automation software. It can also occur by the monitoring of new content published. When new content is published on WordPress, the information is made available on the website pages and also in RSS format, which allows its reading through softwares such as Feedly - a news 
reader. Really Simple Syndication (RSS) is a structured data format packaged by data feeding process [2]. The monitoring of an e-mail software through RSS and the trigger to perform a workflow is equivalent respectively to the functioning of the comparator and activator of cybernetic systems. When a question was classified as answered and published on WordPress, by means of RSS monitoring, the marketing automation software performed a workflow that sent an invitation to site users based on a segmentation for them to answer the question.

In face of a question posted on the website "How can I identify the frequency of posts for each stage of Buyer Journey?", the mere sharing of posts on social networks did not direct a user able to answer the question. On the other hand, the question was not e-mailed since the existing contacts were not yet targeted. The solution was to perform the identification of users on Twitter social network through a search for the keywords "inbound marketing agencies" in the description (bio) of the profile. This identification was limited to agencies located in Brazil, due to the fact that the site was originally published in Portuguese. From the identification of possible users on Twitter, the same open message (tweet) was sent to the profiles that met the search criteria. One of the possible users identified accepted the proposed challenge, answering both the question directly on the website and also the message sent on Twitter. The response was approved and published. Considering the stage of "delivering" and taking the user to his goal, the system sent an e-mail to the user who had asked the question, stating that the answer was available. If the doubt had not been fully clarified, the user could still reply through the website or simply thank it. However, the questioner did not re-interact with the website. As feedback to the user who had answered the question, a new publication on Twitter was made mentioning him and stating that the doubt had been answered.

The final stage of "optimizing" lies in the action-research analysis itself to identify model improvements. One can observe that if the optimization occurred only as the last stage of the PDD, it would have made unfeasible or delayed results that the model proposes. Thus, the optimization phase, common to all three stages, should not occur only at the end of a cycle, as proposed before the action research. It should occur at each stage, for a next stage can only occur if the former is well resolved.

During the action research, it was possible to perform the indirect identification of the persona, as well as the engagement of users directly in social networks, while maintaining spontaneous content creation centralized on the website. Although communication through social networks has proved to be efficient, the use of electronic mail can be considered a form of efficient communication with all users - yet not the only one.

\section{Conclusion}

With the adoption of the proposed PDD model, a content website can benefit from the spontaneous content production by users motivated by their personas - and this production is strengthened through the engagement between them. The Inbound Marketing model consists of four central stages [22]: attract, whose digital marketing actions are designed to attract new visitors to the site; convert, the user identification by content offer; close, in the sense of "closing a deal" by combining digital marketing with commercial actions; and delight, the maintenance of the relationship in after-sales and new purchases. While the delight of buyer personas is the last stage in Inbound and occurs after they become customers, PDD directs people to a playful interaction, prioritizing their experiences at all times of engagement, even before users become customers.

This study aimed to explore the concept of play and its application in a content website as a way to direct its personas to a meaningful playful interaction. Thus, it also sought to define a design model that could be adopted by other designers. Based on the analysis performed, answer to the following question was sought:

"How can you attract the user's attention to a content website, engaging them and leading them to spontaneous content creation?"

- Identifying: each of us has an interest in play manifestations. Seeking such identification is the essence to suit the interests of that persona.

- Engaging: the relationships between people can be motivated and strengthened from the relationship between them and the content of the site itself.

- Delivering: through user feedback it is possible to verify the due improvements in the system, keeping close to the user and informing the perspective and effectiveness of their contribution to the improvement of the website. - Optimizing: the system game tuning is present in all previous stages, so as to enable the system to facilitate the flow state between users.

The Play-Driven Design model suggests its cyclical (iterative) application and an evolutionary perspective of web design projects. When applied in a content website, the proposed model was able to respond to the problematization of the present research, by proposing another interaction approach, and also allowing the spontaneous creation of content. 


\section{Acknowledgments}

This study was carried out by Angelo Públio at the PPGDesign - Stricto Sensu Program on Design -, from the Anhembi Morumbi University, with an academic grant for the Master's Degree thesis "Play-Driven Design: proposal of a model for engagement of personas in content websites" under the supervision of Prof. Dr. Sérgio Nesteriuk.

\section{References}

[1]. MORVILLE, P. Ambient findability: what we find changes who we become. Sebastopol, USA: O'Reilly Media, 2005. 208 p.

[2]. LEVENE, M. An Introduction to Search Engines and Web Navigation. 2.ed. New Jersey: John Wiley \& Sons, 2010.

[3]. CSIKSZENTMIHALYI, M. Creativity: flow and the psychology of discovery and invention. New York: Harper/Collins, 1996, pp. 107126 plus Notes. Available at <http://www.bioenterprise.ca/docs/creativity-by-mihaly-csikszentmihalyi.pdf > Access: January, 23, 2016

[4]. GARRETT, J. The Elements of User Experience: user-centered design for the web and beyond. 2.ed. Berkeley, USA: New Riders, 2011.

[5]. ROLLINGS, A; MORRIS, D. Game Architecture and Design: a new edition. Indianapolis: New Riders, 2004.

[6]. BRANON, R. F.; ESSEX, C. (2001). Synchronous and Asynchronous Communication Tools in Distance Education: a survey of instructors. TechTrends, 45, pp.36-42.

[7]. HUIZINGA, J. Homo Ludens. São Paulo: Perspectiva, 2012.

[8]. SCHELL, J. The Art of Game Design: a book of lenses. Burlington: Elsevier, 2008.

[9]. SALEN, K.; ZIMMERMAN, E. Rules of Play: game design fundamentals. Cambridge, USA: MIT Press, 2003.

[10]. CAILLOIS, R. Os Jogos e os Homens. Lisbon: Cotovia, 1990.

[11]. BARTLE, R. Virtual Worlds: why people play, In: ALEXANDER, T. Massively Multiplayer Game Development. 2.ed. Hingham, USA: Charles River Media, 2005.

[12]. JUUL, J. Half-real: video games between real rules and fictional worlds. Cambridge: MIT Press, 2005.

[13]. BRATHWAITE, B.; SCHREIBER, I. Challenges for Game Designers. Boston: Course Technology, 2009.

[14]. SCHELL, J. The Art of Game Design: a book of lenses. Burlington: Elsevier, 2008.

[15]. CSIKSZENTMIHALYI, M. Flow: the psychology of optimal experience. New York: Harper \& Row, 1990

[16]. ISBISTER, K.; SCHAFFER, N. Game Usability: advancing the player experience. Burlington, EUA: Morgan Kaufman, 2008.

[17]. FULLERTON, T.; SWAIN, C.; HOFFMAN, S. Game Design Workshop: a playcentric approach to creating innovative games. 2.ed. Burlington: Elsevier, 2008.

[18]. COOPER, A. Inmates are Running the Asylum: why high-tech products drive us crazy and how to restore the sanity. Indianapolis, USA: Sams, 2004.

[19]. BARTLE, R. Designing Virtual Worlds. Berkeley, USA: New Riders Publishing, 2004.

[20]. HALLIGAN, B.; SHAH, D. Inbound Marketing: get found using Google, social media, and blogs. Hoboken: John Wiley \& Sons, 2009.

[21]. W3TECHS. Usage of Content Management Systems for Websites. Available at <http://w3techs.com/technologies/overview/content_management/all>. Access: February, 11, 2016.

[22]. YAU, Tina. 2014 HubSpot ROI Report. Cambridge: MIT Sloan School of Management - MBA Class, 2015. 